\title{
BMJ Open Effect of time interval from diagnosis to treatment for non-small cell lung cancer on survival: a national cohort study in Taiwan
}

\author{
Chang-Hung Tsai, ${ }^{1,2}$ Pei-Tseng Kung, ${ }^{3,4}$ Wei-Yin Kuo, ${ }^{5}$ Wen-Chen Tsai (1) ${ }^{5}$
}

To cite: Tsai C-H, Kung PT, Kuo W-Y, et al. Effect of time interval from diagnosis to treatment for nonsmall cell lung cancer on survival: a national cohort study in Taiwan. BMJ Open 2020;10:e034351. doi:10.1136/ bmjopen-2019-034351

- Prepublication history for this paper is available online. To view these files, please visit the journal online (http://dx.doi. org/10.1136/bmjopen-2019034351).

P-TK and W-CT contributed equally.

Received 16 September 2019 Revised 07 March 2020 Accepted 12 March 2020
Check for updates

(C) Author(s) (or their employer(s)) 2020. Re-use permitted under CC BY-NC. No commercial re-use. See rights and permissions. Published by BMJ.

For numbered affiliations see end of article.

Correspondence to Professor Wen-Chen Tsai; wtsai@mail.cmu.edu.tw

\section{ABSTRACT}

Objectives This study aimed to determine if treatment delay after non-small cell lung cancer (NSCLC) diagnosis impacts patient survival rate.

Study design This study is a natural experiment in Taiwan. A retrospective cohort investigation was conducted from 2004 to 2010, which included 42962 patients with newly diagnosed NSCLC.

Methods We identified 42962 patients with newly diagnosed NSCLC in the Taiwan Cancer Registry from 2004 to 2010. We calculated the time interval between diagnosis and treatment initiation. All patients were followed from the index date to death or the end of 2012. Cox proportional hazard models were used to examine the relationship between mortality and time interval.

Results We included 42962 patients (15799 men and 27163 women) with newly diagnosed NSCLC. The mortality rate exhibited a significantly positive correlation to time interval from cancer diagnosis to treatment initiation. The adjusted HRs ranged from 1.04 to 1.08 in all subgroups time interval more than 7 days compared with the counterpart subgroup of the interval from cancer diagnosis to treatment $\leq 7$ days. The trend was also noted regardless of the patients with lung cancer in stage I, stage II and stage III.

Conclusions There is a major association between time to treat and mortality of patients with NSCLC, especially in stages I and II. We suggest that efforts should be made to minimise the interval from diagnosis to treatment while further study is ongoing to determine causation.

\section{INTRODUCTION}

Lung cancer is among the most common causes of cancer death, particularly in industrialised countries. ${ }^{1}$ In addition, the incidence of lung cancer has been gradually increasing over the last 50 years, ${ }^{2}$ becoming a worldwide public health issue. ${ }^{3}$ Lung cancer can be classified into small cell lung cancer and nonsmall cell lung cancer (NSCLC), of which the latter accounts for $80 \%$ of all lung cancer cases. ${ }^{4}$ Despite recent improvements in treatment, the prognosis for patients with lung cancer is still poor. Regardless of whether a patient has NSCLC or small cell lung cancer,
Strengths and limitations of this study

- It consisted of nationwide patients with non-small cell lung cancer.

- We collected nationwide data from 42962 patients with non-small cell lung cancer, which is the largest nationwide study to date.

- There were very few studies investigating treatment delay effects on the reduction of survival rate of patients with lung cancer.

- Our lung cancer stages (stages I, II, III and IV) were based on pathological confirmation.

- Information on patients' quantitative lung function and need for provocative cardiac testing are not available and may be significant factors determining the time to treat interval.

the 5-year survival rate is only $17.4 \% \cdot{ }^{25-7}$ Most lung cancers are in the late stage (stages IIIB or IV) when diagnosed, which is likely to be a result of the long interval between the onset of symptoms and diagnosis. ${ }^{8}$ There are often large differences in the length of the interval from the first appearance of symptoms to confirmation of diagnosis and treatment initiation in these patients. ${ }^{4}$ Although there were many relevant studies on whether differences in the time delay between diagnosis to treatment initiation affects the prognosis of patients with lung cancer, no definitive conclusion has been reached. ${ }^{4}$ An increasing number of relevant studies have also highlighted the importance of this topic.

In Taiwan, $99.68 \%$ of the populace is covered by National Healthcare Insurance (NHI) programme. Under this system, lung cancer is classified as a catastrophic illness and is exempt from treatment-related fees, thereby that patients are not affected by economic factor. According to 2016 statistics from the Taiwan Ministry of Health and Welfare, cancer has consistently been the top cause of death. Among various types of 
cancer, lung cancer ranks first in the cause of death, with a mortality rate of 39.9 per 100000 people. Therefore, it is important for public health providers to improve lung cancer prognoses and increase survival rates. The Taiwan Cancer Registry, a population-based cancer registry, was founded in 1979. The registry is organised by the Ministry of Health and Welfare. The Taiwan Cancer Registry Database records data of all types of cancer diagnosed and treatments in patients in Taiwan. The completeness (97\%) and data quality of the Cancer Registry Database has achieved at an excellent level. ${ }^{9}$ The accuracy of National Health Insurance Research Database (NHIRD) has been validated in previous studies. ${ }^{10}$ This study aims to use national large-scale statistical data to investigate whether the interval between lung cancer diagnosis and treatment affects survival rate; concurrently, we also aim to examine the impact of other relevant factors on survival. This will provide a reference for future treatment for patients with lung cancer of improving their survival.

\section{METHODS}

\section{Data sources and participants}

We included 55014 newly diagnosed patients with NSCLC from 2004 to 2010 . The newly diagnosed patients with NSCLC were defined as International Classification of Diseases for Oncology, 3rd Edition (ICD-O-3) with C339 to C349 without any cancers before. Then we excluded those lung cancer patients with unknown stage for 3993 patients. We also excluded patients with lung cancer in situ (70 patients), with multiple cancer (1298 patients), palliative treatment at the beginning (1934 patients), mortality before lung cancer diagnosed (64 patients), personal characteristics data missing (109 patients) and hospital data missing (4584 patients). In our study, patients with multiple cancers may affect survival due to other cancer effect. In Taiwan, palliative treatment is coded as special code in NHIRD. NSCLC patients with palliative treatment at beginning may be due to patients refusing further treatment or not receiving aggressive treatment. We excluded them for informal treatment. Otherwise, we also excluded those patients with data missing for accuracy. Finally, we had 42962 people.

The data for this study were obtained from the Taiwan Cancer Registry, which was used to acquire study participants. We also linked this data to the National Health Insurance Database and the Cause of Death File from 2002 to 2012 that was provided by the Ministry of Health and Welfare. The accuracy of Taiwan Cancer Registry and NHIRD has achieved at an excellent level.

\section{Patient and public involvement}

No patients were involved in the planning, conception and design of this study, as this study was based on the NHIRD, published by the Ministry of Health and Welfare, Taiwan.

\section{Variable descriptions}

In this study, with regards to the variables used, the general characteristics of patients with lung cancer included sex and age. Age was defined as the age at which the patient had a confirmatory diagnosis by pathology. The financial status of the patient was based on their monthly salary. The degree of urbanisation at the patient's place of residence was used to represent environmental factors. The level of urbanisation was based on seven levels of classification from highly urbanised developed cities (level 1) to remote areas (level 7). The health status of the patient included data on whether the patient had other catastrophic illnesses besides cancer, their Charlson Comorbidity Index (CCI) and the stage of NSCLC. The definition of catastrophic illness was based on the 30 types of catastrophic illnesses or injuries as defined by the National Health Insurance Administration, which include stroke, chronic kidney failure, systemic lupus erythematosus, type I diabetes and severe mental illness. The degree of comorbidity was classified into three levels based on the CCI. ${ }^{11}$ Tumour staging was based on the guidelines of the American Joint Committee on Cancer (6th edition for tumours diagnosed from 2004 to 2009, 7th edition for tumours diagnosed in 2010), which includes stages I, II, III and IV. Hospital attributes include the level of hospital (medical centres, regional hospitals, district hospitals and others), hospital ownership (public or private institutions) and the volume of hospital services (low, medium and high) in treatment of patients with NSCLC. The volume of hospital services was divided into low, medium and high on the basis of quartiles: service volumes of $<25 \%, 25 \%-75 \%$ and $>75 \%$ were defined as low, medium and high, respectively. Patients were considered to be enrolled in multidisciplinary team (MDT) care if they received MDT treatment after pathological diagnosis of NSCLC; the definition of MDT is based on patients who were declared MDT treatment fees in the NHI database (47079B). The interval between diagnosis of NSCLC and treatment initiation was defined as the period between pathological sectioning and diagnosis of NSCLC after biopsy to the time when the patient underwent their first treatment (including surgery, radiotherapy or chemotherapy). The operating definition of relevant treatments is based on the relevant treatment code that was declared in the NHI database, which was checked against the treatment registration information in the Taiwan Cancer Information Database.

\section{Main outcome measurements}

The main outcome examined in this study was the survival rate of patients with lung cancer. Confirmation of death was based on patient data from the NHI database and this was compared with the Taiwan Cause of Death archives for confirmation.

\section{Statistical analysis}

We employed descriptive statistics to show general characteristics, financial status, environmental factors, health status of patients, hospital attributes, enrolment in MDT and the distribution status of the interval from diagnosis confirmation to treatment initiation in patients with lung 
cancer who had a confirmatory diagnosis by pathology from 2004 to 2010. Following this, bivariate analysis was performed using the log-rank test to investigate whether there were significant differences between survival status by the end of 2012 and the interval from diagnosis to treatment initiation. We then used univariate Cox proportional hazards regression to analyse relevant prognostic factors that affect the survival of patients with lung cancer. The adjusted Cox proportional hazards model was used to investigate the relative risk of survival of lung cancer patients with different cancer stages with different intervals from diagnosis confirmation to treatment initiation, after controlling for related variables. Independent variables included patient characteristics, financial status, environmental factors, health status, hospital attributes, enrolment in MDT and grouping of time to treatment initiation. The dependent variable was survival. Lastly, after controlling for relevant variables, the adjusted Cox proportional hazards model was used to generate survival curves for patients with lung cancer of various stages and with different interval periods.

All statistical analyses were performed using SAS software, V.9.2. A p value $<0.05$ was regarded as statistically significant, and all tests were two sided.

\section{RESULTS}

\section{Descriptive statistics of lung cancer patient characteristics} for different treatment intervals

In all patients with lung cancer, the mean 5-year survival rate was $17.61 \%$. As the interval from diagnosis to treatment initiation increased, the 5-year survival rate decreased from $26.12 \%$ to $6.02 \%$ (table 1 ). We also included time to treatment with 0 day $(\mathrm{TTT}=0)$ cases in the $<7$ days group in our study. There were 7363 cases with TTT $=0$ accounting for $17.14 \%$ of all patients in our study. We had 3258 females accounting for $44.25 \%$ in this subgroup. The age of diagnosed in most patients was from 65 to 74 years old accounting for $28.28 \%$ with mean age of 64.67 years old. The treatment of most patients with $\mathrm{TTT}=0$ was surgery combining with radiotherapy and chemotherapy. The 5-year survival rate was $34.9 \%$ in this group with TTT $=0$.

\section{Five-year survival rate of patients for different treatment intervals}

At the same time, we found that in stage I patients, the 5 -year survival rate decreased by $9.07 \%$ due to delayed treatment (patients with intervals $>7$ days). In stage II patients, if patients started treatment earlier (interval $\leq 7$ days), their 5-year survival rate increased by $9.01 \%$. Early treatment (interval $\leq 7$ days) was found to have a smaller effect on 5-year survival rate in stage III and stage IV patients, with an increase in 5-year survival rate of $1.91 \%$ and $0.49 \%$ (table 2).

\section{The effect of different treatment intervals on mortality risk in} patients with lung cancer

Table 3 shows that when the group with interval from cancer diagnosis to treatment $\leq 7$ days was used as a reference, the adjusted HR for mortality in other groups (8-14 days, $15-60$ days and $\geq 61$ days) was significantly increased with increasing interval time (HR 1.04-1.08). Among patients at various cancer stages, using stage I patients as a control group, the adjusted HRs for mortality for cancer patients at various stages was significantly higher (HR 2.06-5.89) and the more advanced the cancer stages, the higher the adjusted HR for mortality. Patients who underwent MDT care had a significantly lower adjusted HR for mortality compared with patients who did not (HR $0.91,95 \%$ CI 0.88 to 0.94 ).

\section{The effect of different treatment intervals on mortality risk in} patients with lung cancer at different cancer stages

Table 4 shows that in patients with stage I lung cancer, with patients with intervals of $\leq 7$ days as the reference group, as the interval increased, the relative risk of death also significantly increased (HR 1.45-2.41). This was also true for stage II (HR 1.21-1.58) and the intervals more than 60 days of patients with stage III lung cancer (HR 1.13, 95\% CI 1.06 to 1.21). In stage IV patients, using patients with an interval $\leq 7$ days as a reference group, the relative risk of death was without significantly difference. Figure 1 shows adjusted survival curve in lung cancer patients with different cancer stages.

\section{DISCUSSION}

We found that the mortality rate exhibited significantly positive correlation to time interval from cancer diagnosis to treatment initiation in NSCLC patients. The ratio of male to female in our study is similar to that in previous studies. ${ }^{12} 13$ The delays between diagnosis and treatment can be categorised into three stages, namely, patient delay, diagnosis delay and treatment delay. ${ }^{2}$ Of all studies that investigated treatment delays in patients with lung cancer, the study with the largest number of patients included 54338 patients but was limited to patients with non-metastatic lung cancer. ${ }^{12}$ In addition, the study only focused on patients who underwent surgical excision and did not analyse the treatment delay status and associated factors for all patients with lung cancer. Another study collected data from 28732 patients to investigate whether treatment delay affects survival rate. ${ }^{14}$ The researchers found that if the interval from diagnosis to treatment was within 35 days, then there is improved survival for patients with localised disease and reduced survival for those with distant disease but did not have significant effect on patients with regional disease. ${ }^{14}$ However, in that study, the diagnosis to treatment interval was divided into just two groups ( $<35$ days and $>35$ days), which makes it more difficult to show the correlation between different treatment delay groups and patient survival rates. In addition, in that study, the authors did not investigate whether different treatment delay periods affect survival rate for different cancer stages as they only classified cancers as localised, regional or distant. To the best of our knowledge, the current study is the first large-scale nationwide 
Table 1 Bivariate analysis of lung cancer patient characteristics for different treatment intervals

Interval from cancer diagnosis to treatment

\begin{tabular}{|c|c|c|c|c|c|c|c|c|c|c|c|}
\hline \multirow{3}{*}{ Variables } & \multirow{2}{*}{\multicolumn{2}{|c|}{ Total }} & \multirow{2}{*}{\multicolumn{2}{|c|}{$\leq 7$ days }} & \multirow{2}{*}{\multicolumn{2}{|c|}{ 8 14 days }} & \multirow{2}{*}{\multicolumn{2}{|c|}{ 15 60 days }} & \multirow{2}{*}{\multicolumn{2}{|c|}{$\geq 61$ days }} & \multirow[b]{3}{*}{$P$ value } \\
\hline & & & & & & & & & & & \\
\hline & $\mathbf{N}$ & $\%$ & $\mathbf{N}$ & $\%$ & $\mathbf{N}$ & $\%$ & $\mathbf{N}$ & $\%$ & $\mathbf{N}$ & $\%$ & \\
\hline Total number & 42962 & 100 & 15769 & 36.7 & 9296 & 21.64 & 12510 & 29.12 & 5387 & 12.54 & - \\
\hline Five-year survival rate & 42962 & 17.61 & 15769 & 26.12 & 9296 & 15.96 & 12510 & 12.99 & 5387 & 6.02 & $<0.001$ \\
\hline \multicolumn{12}{|l|}{ Gender } \\
\hline Female & 15799 & 36.77 & 6154 & 38.95 & 3235 & 20.48 & 4419 & 27.97 & 1991 & 12.6 & \\
\hline Male & 27163 & 63.23 & 9615 & 35.4 & 6061 & 22.31 & 8091 & 29.79 & 3396 & 12.5 & \\
\hline \multicolumn{12}{|l|}{ Age } \\
\hline$\leq 44$ & 2106 & 4.9 & 889 & 42.21 & 455 & 21.6 & 568 & 26.97 & 194 & 9.21 & \\
\hline $45 \sim 54$ & 5686 & 13.23 & 2375 & 41.77 & 1263 & 22.21 & 1549 & 27.24 & 499 & 8.78 & \\
\hline $55 \sim 64$ & 9155 & 21.31 & 3634 & 39.69 & 2033 & 22.21 & 2658 & 29.03 & 830 & 9.07 & \\
\hline $65 \sim 74$ & 12659 & 29.47 & 4548 & 35.93 & 2801 & 22.13 & 3819 & 30.17 & 1491 & 11.78 & \\
\hline$\geq 75$ & 13356 & 31.09 & 4323 & 32.37 & 2744 & 20.55 & 3916 & 29.32 & 2373 & 17.77 & \\
\hline Mean age (m, SD) & 66.76 & 12.44 & 65.52 & 12.55 & 66.45 & 12.22 & 67.04 & 12.15 & 70.25 & 12.46 & $<0.001$ \\
\hline Monthly salary & & & & & & & & & & & $<0.001$ \\
\hline Low-income & 461 & 1.07 & 137 & 29.72 & 101 & 21.91 & 154 & 33.41 & 69 & 14.97 & \\
\hline$\leq 17280$ & 1475 & 3.43 & 542 & 36.75 & 311 & 21.08 & 447 & 30.31 & 175 & 11.86 & \\
\hline $17281 \sim 22800$ & 22935 & 53.38 & 8074 & 35.2 & 5079 & 22.15 & 6751 & 29.44 & 3031 & 13.22 & \\
\hline $22801 \sim 28800$ & 8069 & 18.78 & 2961 & 36.7 & 1690 & 20.94 & 2376 & 29.45 & 1042 & 12.91 & \\
\hline $28801 \sim 36300$ & 2676 & 6.23 & 1011 & 37.78 & 588 & 21.97 & 782 & 29.22 & 295 & 11.02 & \\
\hline $36301 \sim 45800$ & 3280 & 7.63 & 1333 & 40.64 & 689 & 21.01 & 923 & 28.14 & 335 & 10.21 & \\
\hline$\geq 45801$ & 4066 & 9.46 & 1711 & 42.08 & 838 & 20.61 & 1077 & 26.49 & 440 & 10.82 & \\
\hline Urbanisation & & & & & & & & & & & 0.186 \\
\hline Level 1 & 11759 & 27.37 & 4335 & 36.87 & 2494 & 21.21 & 3404 & 28.95 & 1526 & 12.98 & \\
\hline Level 2 & 12117 & 28.2 & 4506 & 37.19 & 2615 & 21.58 & 3527 & 29.11 & 1469 & 12.12 & \\
\hline Level 3 & 6523 & 15.18 & 2334 & 35.78 & 1424 & 21.83 & 1946 & 29.83 & 819 & 12.56 & \\
\hline Level 4 & 6795 & 15.82 & 2518 & 37.06 & 1506 & 22.16 & 1974 & 29.05 & 797 & 11.73 & \\
\hline Level 5 & 1524 & 3.55 & 523 & 34.32 & 338 & 22.18 & 439 & 28.81 & 224 & 14.7 & \\
\hline Level 6 & 2217 & 5.16 & 807 & 36.4 & 490 & 22.1 & 627 & 28.28 & 293 & 13.22 & \\
\hline Level 7 & 2027 & 4.72 & 746 & 36.8 & 429 & 21.16 & 593 & 29.26 & 259 & 12.78 & \\
\hline $\mathrm{CCl}$ score & & & & & & & & & & & $<0.001$ \\
\hline$\leq 3$ & 20388 & 47.46 & 7475 & 36.66 & 4576 & 22.44 & 6186 & 30.34 & 2151 & 10.55 & \\
\hline $4 \sim 6$ & 7587 & 17.66 & 2761 & 36.39 & 1646 & 21.7 & 2218 & 29.23 & 962 & 12.68 & \\
\hline$\geq 7$ & 14987 & 34.88 & 5533 & 36.92 & 3074 & 20.51 & 4106 & 27.4 & 2274 & 15.17 & \\
\hline Other catastrophic illness & & & & & & & & & & & $<0.001$ \\
\hline No & 41474 & 96.54 & 15300 & 36.89 & 8984 & 21.66 & 12076 & 29.12 & 5114 & 12.33 & \\
\hline Yes & 1488 & 3.46 & 469 & 31.52 & 312 & 20.97 & 434 & 29.17 & 273 & 18.35 & \\
\hline Cancer stage & & & & & & & & & & & $<0.001$ \\
\hline Stage I & 5681 & 13.22 & 3226 & 56.79 & 910 & 16.02 & 1269 & 22.34 & 276 & 4.86 & \\
\hline Stage II & 1526 & 3.55 & 589 & 38.6 & 338 & 22.15 & 462 & 30.28 & 137 & 8.98 & \\
\hline Stage III & 11696 & 27.22 & 4030 & 34.46 & 2843 & 24.31 & 3500 & 29.92 & 1323 & 11.31 & \\
\hline Stage IV & 24059 & 56 & 7924 & 32.94 & 5205 & 21.63 & 7279 & 30.25 & 3651 & 15.18 & \\
\hline MDT care & & & & & & & & & & & $<0.001$ \\
\hline No & 37716 & 87.79 & 13669 & 36.24 & 8012 & 21.24 & 10974 & 29.1 & 5061 & 13.42 & \\
\hline Yes & 5246 & 12.21 & 2100 & 40.03 & 1284 & 24.48 & 1536 & 29.28 & 326 & 6.21 & \\
\hline
\end{tabular}

Continued 


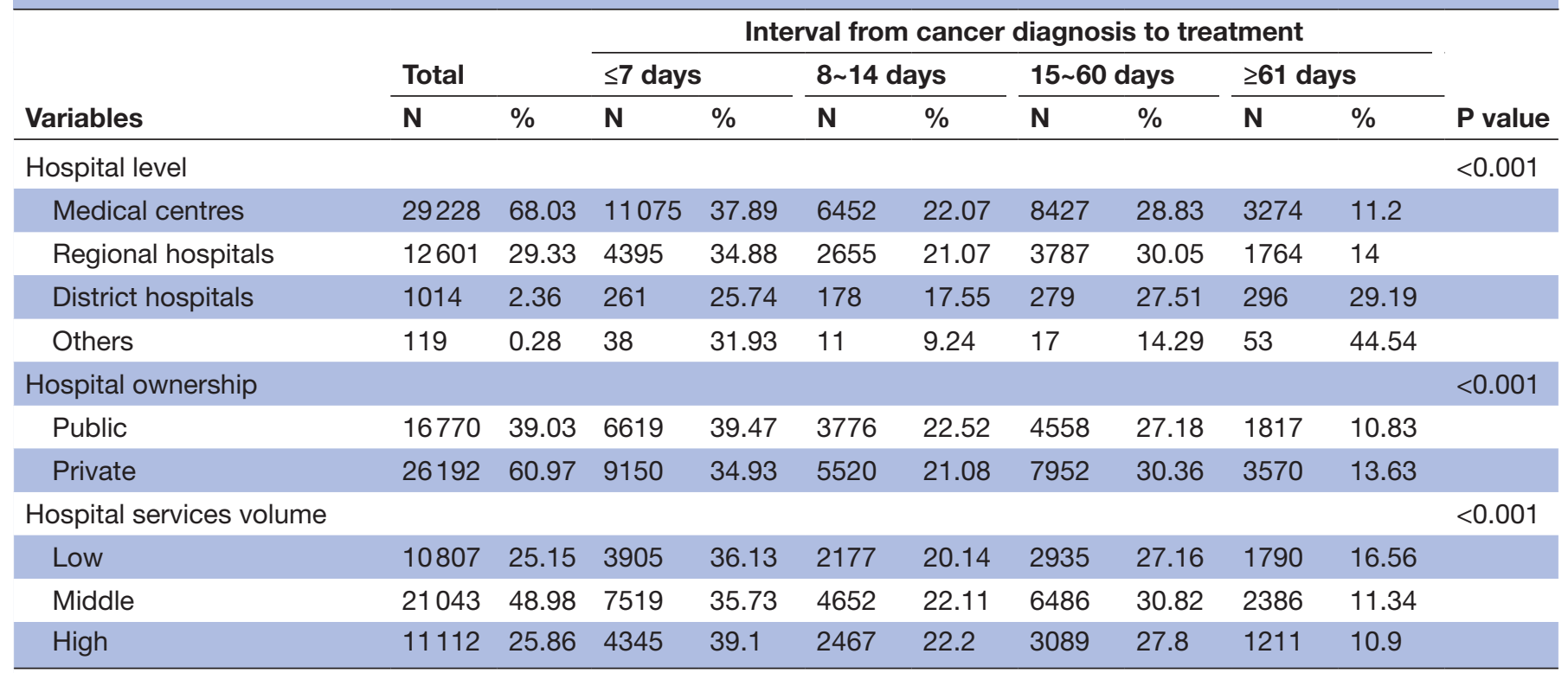

*Log-rank test.

$\mathrm{CCl}$, Charlson Comorbidity Index; MDT, multidisciplinary team.

study that examines whether treatment delay in NSCLC affects patient survival rate. In addition, we also investigated the correlation between lung cancer treatment delay and survival rate for different cancer stages (stages I, II, III and IV).

Previous studies have observed that if patients are older, have more comorbidities or have stage I cancer, they are more likely to delay treatment (interval from diagnosis to treatment $>30$ days). ${ }^{12}$ Similar findings were observed in our study: for patients aged $\geq 55$ years, the greater the age, the greater the proportion with treatment delay (interval $\geq 61$ days) (table 1 ). Patients with high CCI scores also demonstrated significantly increased proportions in treatment delay (interval $\geq 61$ days) (table 1 ). CCI is a general score to evaluate patients' comorbidity and does not focus on patients with lung cancer. The lung function testing such as forced expiratory volume in $1 \mathrm{~s}\left(\mathrm{FEV}_{1}\right)$ and diffusing capacity of the lung for carbon monoxide (DLCO) is more accurate for evaluating their severity but is not available in our study. It is a fact that patients with poorer lung function require additional testing to determine candidacy for surgery. This testing, including $6 \mathrm{~min}$ walk test, quantitative perfusion scans, cardiopulmonary exercise testing and consultation with pulmonary medicine takes time and is not available in our study. However, during analysis of the correlation between treatment delay and lung cancer stage, we found that the proportion of stage I patients with treatment delay was significantly lower than patients with other stages of lung cancer. A previous study has observed that in patients with NSCLC, treatment delay is not associated with cancer stage. ${ }^{15-20} \mathrm{In}$ contrast, treatment delay had more serious effects in stage III and IV patients. ${ }^{21}$ However, in our study, we found that the proportion of stage I patients with treatment delay (interval from diagnosis to treatment $\geq 61$ days) was significantly lower $(4.86 \%, \mathrm{p}<0.001)$ when compared with patients at other stages.

Previous studies have mentioned that in patients with NSCLC, our understanding of the effects of diagnosis and treatment delay on the prognosis of patients is

Table 2 Five-year survival rate of patients for different treatment intervals

\begin{tabular}{|c|c|c|c|c|c|c|c|c|c|}
\hline \multirow[b]{2}{*}{ Variables } & \multicolumn{2}{|c|}{ Stage I } & \multicolumn{2}{|c|}{ Stage II } & \multicolumn{2}{|c|}{ Stage III } & \multicolumn{2}{|c|}{ Stage IV } & \multirow[b]{2}{*}{$P$ value * } \\
\hline & $\mathbf{N}$ & $\%$ & $\mathbf{N}$ & $\%$ & $\mathbf{N}$ & $\%$ & $\mathbf{N}$ & $\%$ & \\
\hline Total number & 5681 & 61.61 & 1526 & 34.41 & 11696 & 12.95 & 24059 & 5.11 & \\
\hline$\leq 7$ days & 3226 & 70.68 & 589 & 43.42 & 4030 & 14.86 & 7924 & 5.60 & \\
\hline 8 14 days & 910 & 60.58 & 338 & 33.74 & 2843 & 12.11 & 5205 & 4.58 & \\
\hline$\geq 61$ days & 276 & 21.10 & 137 & 14.56 & 1323 & 6.83 & 3651 & 4.12 & \\
\hline
\end{tabular}

*Log-rank test. 
Table 3 Relative risk of death in patients for different treatment intervals

\section{Unadjusted}

\section{Variables}

HR P value

Adjusted

HR $95 \% \mathrm{Cl}$

P value*

Interval from cancer diagnosis to treatment $\leq 7$ days (ref.)

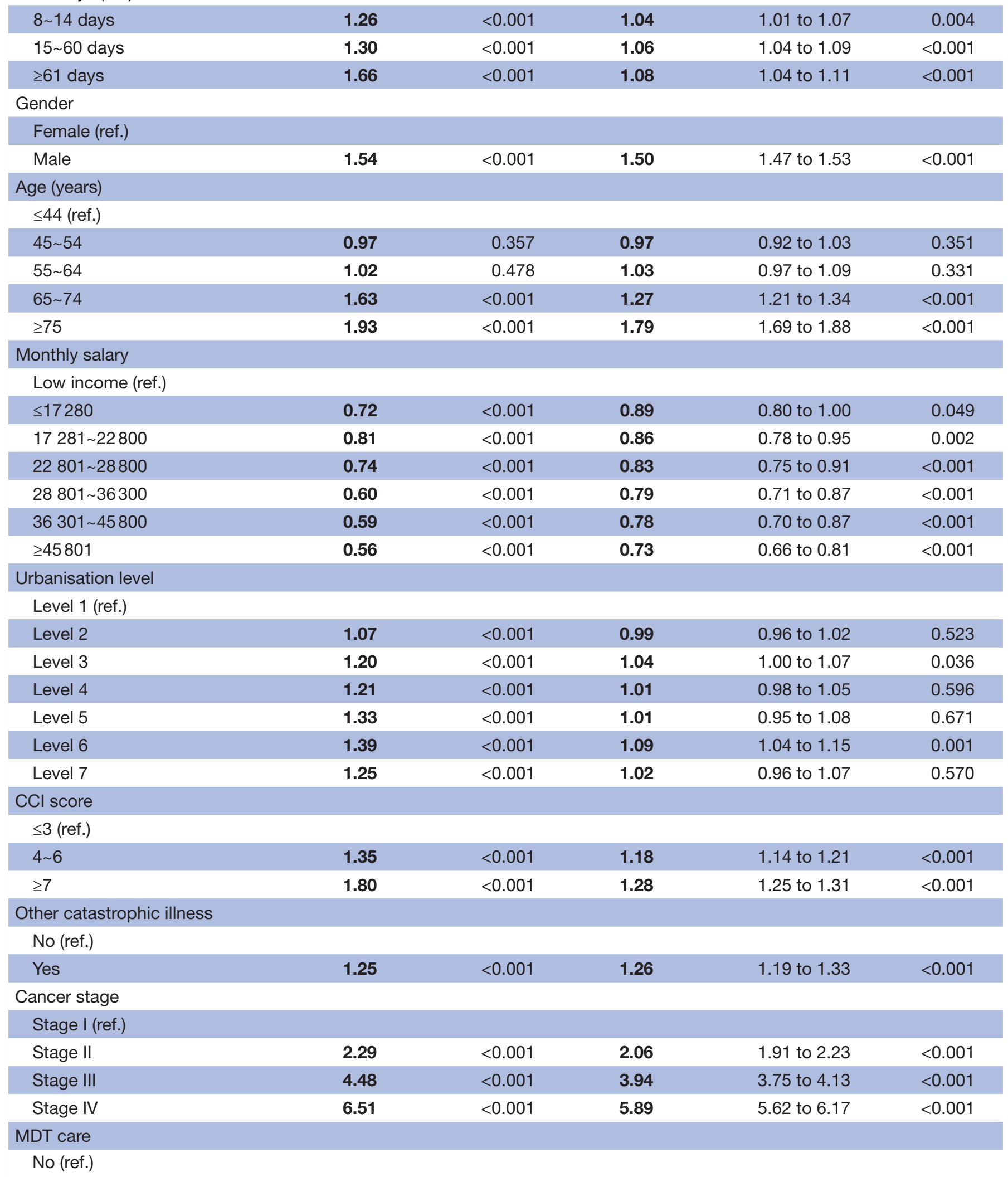

Continued 
Table 3 Continued

\begin{tabular}{|c|c|c|c|c|c|}
\hline \multirow[b]{2}{*}{ Variables } & \multicolumn{2}{|c|}{ Unadjusted } & \multicolumn{3}{|c|}{ Adjusted } \\
\hline & HR & $P$ value & HR & $95 \% \mathrm{Cl}$ & P value* \\
\hline Yes & 0.95 & 0.001 & 0.91 & 0.88 to 0.94 & $<0.001$ \\
\hline \multicolumn{6}{|l|}{ Hospital level } \\
\hline \multicolumn{6}{|c|}{ Medical centres (ref.) } \\
\hline Regional hospitals & 1.28 & $<0.001$ & 0.99 & 0.96 to 1.02 & 0.347 \\
\hline District hospitals & 2.06 & $<0.001$ & 1.25 & 1.17 to 1.34 & $<0.001$ \\
\hline Others & 1.17 & 0.137 & 0.90 & 0.73 to 1.10 & 0.286 \\
\hline \multicolumn{6}{|l|}{ Hospital ownership } \\
\hline \multicolumn{6}{|l|}{ Public (ref.) } \\
\hline Private & 1.27 & $<0.001$ & 1.13 & 1.10 to 1.16 & $<0.001$ \\
\hline \multicolumn{6}{|c|}{ Hospital services volume } \\
\hline \multicolumn{6}{|l|}{ Low (ref.) } \\
\hline Middle & 0.72 & $<0.001$ & 0.83 & 0.81 to 0.85 & $<0.001$ \\
\hline High & 0.59 & $<0.001$ & 0.71 & 0.68 to 0.74 & $<0.001$ \\
\hline
\end{tabular}

${ }^{*}$ Cox proportional hazards regression.

$\mathrm{CCl}$, Charlson Comorbidity Index; MDT, multidisciplinary team.

limited, although an increasing number of recent studies are emphasising the importance of this topic. ${ }^{4}$ Some studies have found that in patients with a symptom-totreatment interval (STI) of $>60$ days, the survival rate was significantly higher than that of patients with a STI of $<60$ days. ${ }^{22}$ However, if patients were further divided on the basis of the type of lung cancer, this difference was only significant in patients with NSCLC. ${ }^{22}$ However, the number of patients included in this study was only 103 (96 men). ${ }^{22}$ Two other studies, with 378 and 410 patients each, found that delaying diagnosis and treatment did not affect patient survival rates. ${ }^{16}{ }^{17}$ Another study of 466 patients with NSCLC found that patients with shorter STIs had lower survival rates. ${ }^{23}$ One study with 189 patients with lung cancer found that treatment delay resulted in poorer prognosis for patients, ${ }^{24}$ while another study with 132 patients found that longer specialist treatment delay does not result in poorer prognosis. ${ }^{25}$ An aforementioned article also observed that most previous studies in different countries were monocentric studies and that it is difficult to decide which study is most reliable with regards to whether treatment delay affects patient survival rates. $^{4}$

Most studies show no relationship between time-tochemotherapy and their survival rate. ${ }^{26}$ However, it should be noted that in these review articles, the number of cases collected is generally very low, with the highest number of patients only $10583 .^{26}$ Another study showed time intervals from diagnosis to treatment were not associated with survival outcomes in NSCLC. ${ }^{27}$ In this previous study, they discussed NSCLC patients with different treatment such as surgery, radiotherapy, systemic therapy and palliative care, which were not discussed in our study. They also suggested that delays to treatment might impact on

Table 4 Relative risk of death in patients for different treatment intervals and at different cancer stages

\begin{tabular}{|c|c|c|c|c|c|c|c|c|c|c|c|c|c|c|c|}
\hline \multirow[b]{2}{*}{ Variables } & \multicolumn{3}{|c|}{ Stage $I^{*}$} & \multicolumn{4}{|c|}{ Stage II* } & \multicolumn{4}{|c|}{ Stage III* } & \multicolumn{4}{|c|}{ Stage IV* } \\
\hline & HR & $95 \% \mathrm{Cl}$ & $\begin{array}{l}\mathbf{P} \\
\text { valuet }\end{array}$ & HR & $95 \%$ & & $\begin{array}{l}P \\
\text { valuet }\end{array}$ & HR & $95 \% \mathrm{C}$ & & $\begin{array}{l}P \\
\text { valuet }\end{array}$ & HR & $95 \% \mathrm{C}$ & & $\begin{array}{l}P \\
\text { value }\end{array}$ \\
\hline \multicolumn{16}{|c|}{ Interval from cancer diagnosis to treatment } \\
\hline \multicolumn{16}{|c|}{$\leq 7$ days (ref.) } \\
\hline $8 \sim 14$ days & 1.45 & 1.281 .64 & $<0.001$ & 1.21 & 1.01 & 1.45 & 0.039 & 1.04 & 0.98 & 1.09 & 0.177 & 0.99 & 0.95 & 1.03 & 0.561 \\
\hline $\begin{array}{l}15 \sim 60 \\
\text { days }\end{array}$ & 1.66 & 1.491 .84 & $<0.001$ & 1.44 & 1.22 & 1.69 & $<0.001$ & 1.02 & 0.97 & 1.07 & 0.560 & 1.01 & 0.98 & 1.04 & 0.572 \\
\hline$\geq 61$ days & 2.41 & $2.06 \quad 2.83$ & $<0.001$ & 1.58 & 1.26 & 1.97 & $<0.001$ & 1.13 & 1.06 & 1.21 & $<0.001$ & 0.98 & 0.94 & 1.02 & 0.249 \\
\hline
\end{tabular}

${ }^{*}$ Patient age, gender, monthly salary, level of urbanisation of place of residence, CCl score, other illness, MDT care, hospital level, hospital ownership and hospital services volume were all controlled for using various models.

†Adjusted Cox proportional hazards model.

$\mathrm{CCl}$, Charlson Comorbidity Index; MDT, multidisciplinary team. 

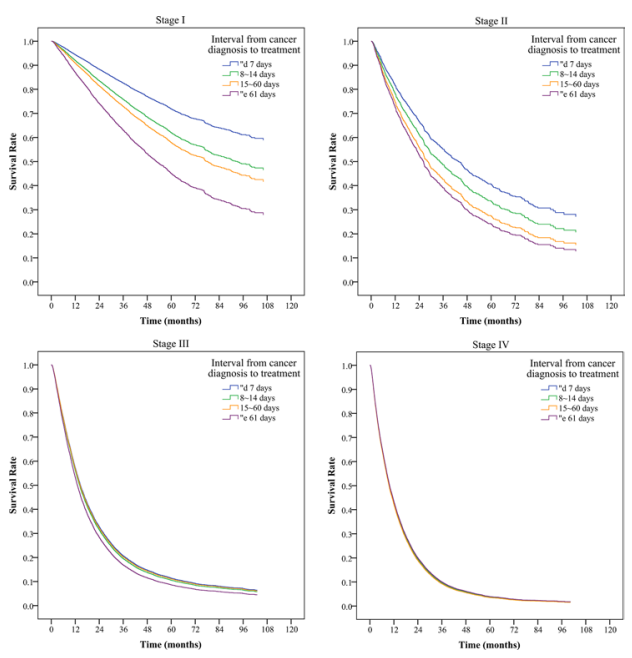

Figure 1 Adjusted survival curve in lung cancer patients with different cancer stages.

other outcomes other than survival. However, there were only 1729 patients in this previous study. ${ }^{27}$ In summary, the majority of previous studies into whether treatment delay affects survival rate in patients with NSCLC lack large-scale nationwide statistical data. This can easily lead to bias and produce divergent conclusions. In this study, we collected nationwide data from 42962 patients with NSCLC which, to the best of our knowledge, is the largest nationwide study to date. In addition, we also investigated the correlation between lung cancer treatment delay and survival rate in different cancer stages (stages I, II, III and IV).

In addition, detailed examination of the literature found that a decreased treatment delay increases the risk of death in patients; the explanation provided for this is that a shorter treatment delay may mean that the patients have more obvious or more severe symptoms. ${ }^{23}$ Therefore, there is a need to correct the result with cancer stage and severity. ${ }^{23}$ A previous study has also suggested that a shorter treatment delay may reflect a requirement for more urgent treatment due to severity of symptoms, resulting in a poor prognosis. ${ }^{28}$ Therefore, in this paper, we also considered the effects of cancer stage on treatment delay and patient prognosis. In another paper, it was also mentioned that the definition of treatment delay should be more standardised and accurate. ${ }^{4}$ Another paper mentioned that it is not easy to accurately calculate the time of treatment initiation. ${ }^{23}$ In addition, the calculation of patient delay (from symptom to doctor) is also prone to errors. Therefore, in this study, our definition of treatment delay was made according to the cancer registration archives and NHI database, from pathological diagnosis confirmation until treatment initiation.

For patients with cancer in general, current medical guidelines all recommend early diagnosis and treatment to improve patient prognosis. However, early diagnosis is difficult due to multiple factors, such as nonapparent symptoms and patient delay. However, in this study, we found that if the interval from confirmation of pathological diagnosis to treatment initiation in patients with NSCLC is shortened to 7 days, this is associated with an improvement in 5-year life expectancy (improvements of $0.49 \%-9.07 \%$ were observed, according to the different stages of lung cancer). We also found that this improvement in 5-year survival rate was particularly marked for patients with NSCLC at early stages (stage I and II), at $10.28 \%-10.34 \%$. However, in late stage (stage III and stage IV) patients, the 5-year survival rate was only increased by $1.91 \%$ and $0.49 \%$. It is extremely ambitious for lung cancer treatment to commence within 7 days of diagnosis considering the staging exam taking time. This group in the study ( $<7$ days to treatment) may be skewed towards those whose cancer was diagnosed at the time or surgery. A previous study showed that NSCLC growth rate appeared to be highly variable and related to histological subtype, which was not discussed in our study. ${ }^{29}$ Doubling times can be quite variable in different stages of NSCLC. Another study showed that rapid tumour progression was noted in patients with untreated, predominantly stage III NSCLC. ${ }^{30}$ In our study, table 4 shows stage III NSCLC patients with the interval from diagnosis to treatment initiation more than 60 days had significantly higher relative risk of death than patients with an interval $\leq 7$ days (HR 1.13, 95\% CI 1.06 to 1.21). This may be due to rapid tumour progression characteristics of stage III NSCLC. However, the delay treatment effect is not significant in patients with stage IV NSCLC, which may be associated with poor outcome and low survival rate in late stage of NSCLC. Therefore, we recommended that in future policies, treatment recommendations should be formulated so that patients can start treatment within 7 days after pathological diagnosis confirmation of NSCLC to increase their 5-year survival rate. This is particularly important for patients with early stage (stage I and II) NSCLC, where improvement effects are more significant.

In this study, we also found that the effect of the interval from diagnosis to treatment initiation and patient survival rate decreased with more advanced cancer stages. Therefore, in patients who have stage I (HR 1.45-2.41) or stage II (HR 1.21-1.58) cancer, the longer the interval from diagnosis to treatment initiation, the higher the risk of death in patients. However, in stage III patients, compared with patients with an interval from diagnosis to treatment initiation $\leq 7$ days, only when the interval from diagnosis to treatment initiation was $\geq 61$ days was the risk of death increased. However, the magnitude of the increased risk of death is lower than in stage I and II patients (HR 1.03-1.06). It appears the association between time to treatment and outcome is stronger with lower stage disease. This may have implications on resource allocation specifically addressing the time to treatment (TTT) phenomenon. Further study, however, is necessary to better understand causation.

In recent studies, it was found that patients with oral cancers who underwent MDT treatment had significantly higher survival rates and that the proportion of patients who underwent treatment was higher than those who 
did not join MDT. ${ }^{31}$ Previous studies have shown that the use of MDT care in cancer treatment can improve patient prognosis. ${ }^{32}$ This is particularly the case in head and neck cancers, where MDT care is cost-effective and can improve survival rates. ${ }^{33}$ Previous studies have shown that in patients with lung cancer MDT care can significantly improve the patient's acceptance of treatment but does not significantly improve patient survival rates. ${ }^{32}$ In this study, we found that patients who underwent MDT care had a significantly lower adjusted HR for mortality compared with patients who did not (HR 0.91, 95\% CI 0.88 to 0.94 ).

In summary, this study identifies an association between time to treat and survival in NSCLC. Although causation is not definitive, efforts to diminish time to treat in the patient with lung cancer would seem prudent while awaiting further study on the issue. In addition, in patients with NSCLC, we recommend the addition of MDT care to decrease the risk of death and improve prognosis.

\section{Limitations}

A secondary random database derived from the NHIRD was employed for this study. The information on individual lifestyle and health behaviours, which may also affect the result, is not available. The lung function testing such as $\mathrm{FEV}_{1}$ and DLCO is not available in our database, and disease-free survival is also not available in our study.

\section{CONCLUSIONS}

In this study, we collected nationwide data from 42962 patients with NSCLC which, to the best of our knowledge, is the largest nationwide study to date. In addition, we also investigated the correlation between lung cancer treatment delay and survival rate in different cancer stages (stages I, II, III and stage IV) with pathological confirmation. Treatment timeliness is associated with better survival rates in patients with NSCLC, particularly stages I and II.

\section{Author affiliations}

${ }^{1}$ Department of Public Health, China Medical University, Taichung, Taiwan ${ }^{2}$ Department of Emergency Medicine, Miaoli General Hospital Ministry of Health and Welfare, Miaoli, Taiwan

${ }^{3}$ Department of Healthcare Administration, Asia University, Taichung, Taiwan ${ }^{4}$ Department of Medical Research, China Medical University Hospital, China Medical University, Taichung, Taiwan

${ }^{5}$ Department of Health Services Administration, China Medical University, Taichung, Taiwan

Acknowledgements We are grateful to the Science Centre of the Ministry of Health and Welfare for providing us with access to the National Health Insurance Research Database, Cancer Registry Files and Cause of Death File. We are also grateful to Health Data Science Centre, China Medical University Hospital for providing administrative, technical and funding support.

Contributors Conceptualisation: C-HT, P-TK and W-CT. Methodology: all authors. Software: W-CT. Validation: C-HT, P-TK and W-CT. Formal analysis: C-HT and W-YK. Data curation: C-HT, P-TK and W-CT. Resources: P-TK and W-CT. Writing (original draft preparation): C-HT and W-C T. Writing (review and editing): C-HT, P-TK and W-CT. Supervision: W-CT. Project administration: C-HT, W-YK and P-TK. Funding acquisition: P-TK and W-CT.
Funding This study was supported by the grants (CMU107-ASIA-18; MOST 104-2410 H-039 -002) from China Medical University, Asia University and the Ministry of Science and Technology, Taiwan.

Competing interests None declared.

Patient and public involvement Patients and/or the public were not involved in the design, or conduct, or reporting, or dissemination plans of this research.

Patient consent for publication Not required.

Provenance and peer review Not commissioned; externally peer reviewed.

Data availability statement No data are available. This study used the National Health Insurance Research Database published by the Ministry of Health, Taiwan. Due to legal restrictions imposed by the Taiwan government related to the Personal Information Protection Act, the database cannot be made publicly available. All researchers can apply for using the databases for conducting their studies. Requests for data can be sent as a formal proposal to the Science Center of the Ministry of Health and Welfare (http://www.mohw.gov.tw/EN/Ministry/Index.aspx). Any raw data are not allowed to be brought out from the Science Center. Only the analytic outputs in format of table or figure can be printed out. The restrictions prohibited the authors from making the minimal data set publicly available.

Open access This is an open access article distributed in accordance with the Creative Commons Attribution Non Commercial (CC BY-NC 4.0) license, which permits others to distribute, remix, adapt, build upon this work non-commercially, and license their derivative works on different terms, provided the original work is properly cited, appropriate credit is given, any changes made indicated, and the use is non-commercial. See: http://creativecommons.org/licenses/by-nc/4.0/.

ORCID iD

Wen-Chen Tsai http://orcid.org/0000-0002-9684-0789

\section{REFERENCES}

1 Ferlay J, Shin H-R, Bray F, et al. Estimates of worldwide burden of cancer in 2008: GLOBOCAN 2008. Int J Cancer 2010;127:2893-917.

2 Jensen AR, Mainz J, Overgaard J. Impact of delay on diagnosis and treatment of primary lung cancer. Acta Oncol 2002;41:147-52.

3 Valdés S, García E, Pérez H, et al. Length of diagnostic delay in patients with non-small-cell lung cancer. MEDICC Rev 2010;12:2932.

4 Vinas F, Ben Hassen I, Jabot L, et al. Delays for diagnosis and treatment of lung cancers: a systematic review. Clin Respir J 2016;10:267-71.

5 Storm HH, Dickman PW, Engeland A, et al. Do morphology and stage explain the inferior lung cancer survival in Denmark? Eur Respir J 1999;13:430-5.

6 Teppo L, Dickman PW, Hakulinen T, et al. Cancer patient survival-patterns, comparisons, trends--a population-based Cancer Registry study in Finland. Acta Oncol 1999;38:283-94.

7 Ettinger DS, Wood DE, Akerley W, et al. NCCN guidelines insights: non-small cell lung cancer, version 4.2016. J Natl Compr Canc Netw 2016;14:255-64.

8 Chandra S, Mohan A, Guleria R, et al. Delays during the diagnostic evaluation and treatment of lung cancer. Asian Pac J Cancer Prev 2009;10:453-6.

9 Chiang C-J, You S-L, Chen C-J, et al. Quality assessment and improvement of nationwide cancer registration system in Taiwan: a review. Jpn J Clin Oncol 2015;45:291-6.

10 Cheng C-L, Kao Y-HY, Lin S-J, et al. Validation of the National health insurance research database with ischemic stroke cases in Taiwan. Pharmacoepidemiol Drug Saf 2011;20:236-42.

11 Deyo RA, Cherkin DC, Ciol MA. Adapting a clinical comorbidity index for use with ICD-9-CM administrative databases. J Clin Epidemiol 1992;45:613-9.

12 Bilimoria KY, Ko CY, Tomlinson JS, et al. Wait times for cancer surgery in the United States: trends and predictors of delays. Ann Surg 2011;253:779-85.

13 Evans SM, Earnest A, Bower W, et al. Timeliness of lung cancer care in Victoria: a retrospective cohort study. Med J Aust 2016;204:75.

14 Gomez DR, Liao K-P, Swisher SG, et al. Time to treatment as a quality metric in lung cancer: staging studies, time to treatment, and patient survival. Radiother Oncol 2015;115:257-63.

15 Liberman M, Liberman D, Sampalis JS, et al. Delays to surgery in non-small-cell lung cancer. Can J Surg 2006;49:31.

16 Pita-Fernández S, Montero-Martinez C, Pértega-Diaz S, et al. Relationship between delayed diagnosis and the degree of invasion and survival in lung cancer. J Clin Epidemiol 2003;56:820-5. 
17 Porta M, Gallén M, Malats N, et al. Influence of "diagnostic delay" upon cancer survival: an analysis of five tumour sites. J Epidemiol Community Health 1991;45:225-30.

18 Koyi H, Hillerdal G, Brandén E. Patient's and doctors' delays in the diagnosis of chest tumors. Lung Cancer 2002;35:53-7.

19 Billing JS, Wells FC. Delays in the diagnosis and surgical treatment of lung cancer. Thorax 1996;51:903-6.

20 Mor V, Masterson-Allen S, Goldberg R, et al. Pre-Diagnostic symptom recognition and help seeking among cancer patients. $J$ Community Health 1990;15:253-66.

21 Christensen ED, Harvald T, Jendresen M, et al. The impact of delayed diagnosis of lung cancer on the stage at the time of operation. Eur $J$ Cardiothorac Surg 1997;12:880-4.

22 Annakkaya AN, Arbak P, Balbay O, et al. Effect of symptom-totreatment interval on prognosis in lung cancer. Tumori 2007;93:61-7.

23 Myrdal G, Lambe M, Hillerdal G, et al. Effect of delays on prognosis in patients with non-small cell lung cancer. Thorax 2004;59:45-9.

24 Robinson E, Mohilever J, Zidan J, et al. Delay in diagnosis of cancer. Possible effects on the stage of disease and survival. Cancer 1984;54:1454-60.

25 Salomaa E-R, Sällinen S, Hiekkanen H, et al. Delays in the diagnosis and treatment of lung cancer. Chest 2005;128:2282-8.

26 Alexander M, Blum R, Burbury K, et al. Timely initiation of chemotherapy: a systematic literature review of six priority cancers - results and recommendations for clinical practice. Intern Med J 2017;47:16-34.
27 Vinod SK, Chandra A, Berthelsen A, et al. Does timeliness of care in non-small cell lung cancer impact on survival? Lung Cancer 2017;112:16-24.

28 Diaconescu R, Lafond C, Whittom R. Treatment delays in non-small cell lung cancer and their prognostic implications. J Thorac Oncol 2011;6:1254-9.

29 Mackintosh JA, Marshall HM, Yang IA, et al. A retrospective study of volume doubling time in surgically resected non-small cell lung cancer. Respirology 2014;19:755-62.

30 Everitt S, Herschtal A, Callahan J, et al. High rates of tumor growth and disease progression detected on serial pretreatment fluorodeoxyglucose-positron emission tomography/computed tomography scans in radical radiotherapy candidates with nonsmall cell lung cancer. Cancer 2010;116:5030-7.

31 Tsai W-C, Kung P-T, Wang S-T, et al. Beneficial impact of multidisciplinary team management on the survival in different stages of oral cavity cancer patients: results of a nationwide cohort study in Taiwan. Oral Oncol 2015;51:105-11.

32 Boxer MM, Vinod SK, Shafiq J, et al. Do multidisciplinary team meetings make a difference in the management of lung cancer? Cancer 2011;117:5112-20.

33 Birchall M, Bailey D, King P, et al. Effect of process standards on survival of patients with head and neck cancer in the South and West of England. Br J Cancer 2004;91:1477-81. 